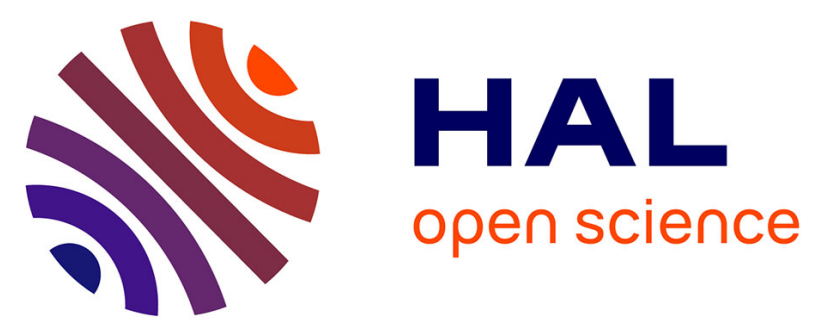

\title{
On The Energy Efficiency of Hybrid Unicast-Broadcast Networks for Mobile TV Services
}

Pape Abdoulaye Fam, Stéphane Paquelet, Matthieu Crussière, Jean-François Hélard, Pierre Brétillon

\section{> To cite this version:}

Pape Abdoulaye Fam, Stéphane Paquelet, Matthieu Crussière, Jean-François Hélard, Pierre Brétillon. On The Energy Efficiency of Hybrid Unicast-Broadcast Networks for Mobile TV Services. Journée Scientifique URSI-France 2016 Energie et Radiosciences, Mar 2016, Rennes, France. hal-01290493

\section{HAL Id: hal-01290493 \\ https://hal.science/hal-01290493}

Submitted on 18 Mar 2016

HAL is a multi-disciplinary open access archive for the deposit and dissemination of scientific research documents, whether they are published or not. The documents may come from teaching and research institutions in France or abroad, or from public or private research centers.
L'archive ouverte pluridisciplinaire HAL, est destinée au dépôt et à la diffusion de documents scientifiques de niveau recherche, publiés ou non, émanant des établissements d'enseignement et de recherche français ou étrangers, des laboratoires publics ou privés. 


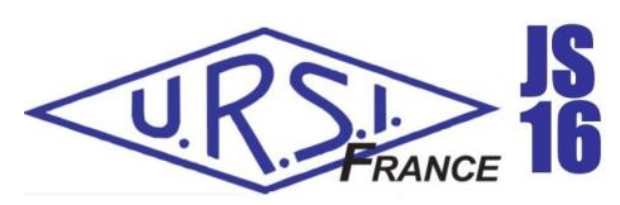

\title{
Energy and Radio Science
}

\section{On The Energy Efficiency of Hybrid Unicast-Broadcast Networks for Mobile TV Services}

\author{
Pape Abdoulaye Fam*, Stéphane Paquelet*, Matthieu Crussière**, Jean-François Hélard**, Pierre Brétillon *** \\ *b<>com, Rennes, France. pape-abdoulaye.fam@b-com.com, \\ ** INSA-IETR, Rennes, France. \\ *** TDF, Metz, France.
}

Mots clés (Keywords): Convergence des réseaux broadband et broadcast (Convergence of broadcasting and broadband wireless), Couverture et efficacité énergétique (Coverage and energy efficiency).

\begin{abstract}
An exponential growth of the mobile data traffic is expected in future networks. The reason of this growth is related to the increasing popularity of linear services such as mobile TV, live and sports events, which may lead to the delivery of the same contents to a large audience. With this mobile data traffic explosion, the power demand of future wireless networks is predicted to dramatically increase. This energy consumption issue, if unaddressed, may lead to the restriction of many new services. Based on these facts, recent studies have shown that network cooperation is a promising candidate to deal with such issues. This paper investigates, from an energy efficiency point of view, the performances of using a hybrid unicast/broadcast network to deliver linear services to mobile and portable devices. Results show that cooperation will decrease the total power consumption of the hybrid network.
\end{abstract}

\section{Introduction}

In the last few years, many studies in the literature have addressed the cooperation between broadcast and unicast networks. On the one hand, first studies have raised the question of network selection assuming a common coverage area for both broadcast and unicast networks [1]-[3]. On the other hand, the network cooperation topic has been studied from a planning perspective point of view, as in [4], [5], by considering different coverage areas for the two networks. It has then been shown that the coverage extension of the service area is the optimal cooperation strategy to improve the network efficiency in terms of capacity, energy consumption and quality of service. However these latter studies assume a broadcast transmission for the two networks. Therefore, we have recently investigated the service coverage extension scenario considering a broadcast transmission mode for the broadcast network and a unicast transmission mode for the unicast network in [6]. The proposed hybrid network consists of two Orthogonal Frequency Division Multiplexing (OFDM) systems: a broadcast network composed of a single High Power High Tower (HPHT) station delivering a broadcast signal such as DVB-NGH/T2Lite or a modified version of the LTE broadcast signal (eMBMS) and a unicast network composed of several Low Power Low Tower (LPLT) sites producing a unicast signal according to the LTE standard. Hereby, we have introduced an analytical model to provide the optimal coverage area of the broadcast network that maximizes the overall capacity for a given service area. This present paper is an extension of our previous work. In this paper, the performances of the hybrid network are discussed from an energy efficiency point of view. First, based on the research project EARTH [7], we have enhanced the hybrid model proposed in [6] by adding a power consumption metric to the model. We then show by simulation that increasing the overall capacity of the hybrid network reduces the total power consumption in terms of total power per bit used to deliver the proposed service in a given service area.

\section{Power Consumption Model}

The energy consumption of wireless network has been widely investigated in the literature. For instance, it is shown in [7], [8], that base stations represent a dominant share of the total power consumption in mobile broadband and broadcast networks. Furthermore, with the explosion of wireless communications systems, the reduction of network power consumption becomes more and more critical [8]. Therefore a simple and accurate model is proposed in [7] to evaluate the base station power consumption and assess the performances of power reduction techniques. 
The proposed power consumption model is based on a combination of base station components and sub-components such as analog Radio Frequency (RF), baseband (BB) processing, and power amplifier and the power system (cooling).

Based on the research project EARTH findings [7], the power consumption $P_{\text {in }}$ of a base station is given by

$$
P_{\text {in }}=N_{T R X} \frac{\frac{P_{\text {out }}}{\eta_{P A}\left(1-\sigma_{\text {feed }}\right)}+P_{R F}+P_{B B}}{\left(1-\sigma_{D C}\right)\left(1-\sigma_{M S}\right)\left(1-\sigma_{\text {cool }}\right)},
$$

where $N_{T R X}$ is the number of transceiver chains at the base station, $\eta_{P A}$ is the efficiency of the power amplifier, $\sigma_{f e e d}$ represents the feeder losses, and $P_{\text {out }}=\rho P_{\text {max }}$ is the power consumption of a single transceiver chains depending on its load $\rho$ and the maximum power $P_{\max }$ available at the transmitter. $P_{R F}$ and $P_{B B}$ are respectively the power consumption of the RF transceiver and the baseband processing modules. Finally, $\sigma_{D C}, \sigma_{M S}, \sigma_{c o o l}$ refer to the loss factors of the DC-DC power supply, the main supply and active cooling power consumption. This power consumption model is used to evaluate the network power consumption in the following.

\section{Hybrid Network Model}

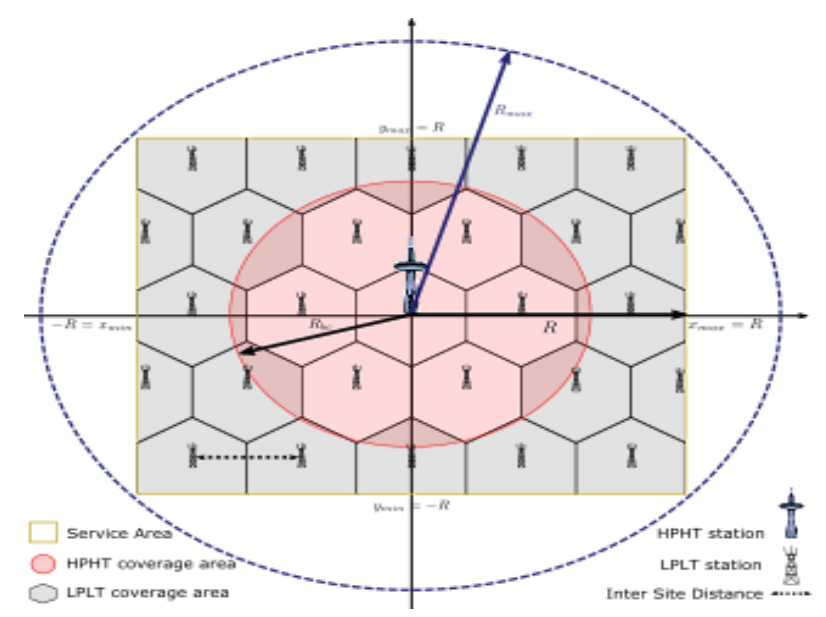

Fig. 1- Hybrid network model

The proposed hybrid network model is described in Fig. 1. As shown in Fig. 1, the hybrid network consists of two Orthogonal Frequency Division Multiplexing (OFDM) systems: a broadcasting system composed of a single High Power High Tower (HPHT) site located at the center of the service area and a mobile broadband system composed of $N_{L P L T}$ Low Power Low Tower (LPLT) sites producing a unicast signal according to the LTE standard. The two systems operate in two different frequency bands, without any mutual interference.

Let us consider a transmission of a linear TV service to $M$ users uniformly distributed in a given service area. It is assumed that the service is always available and can be continuously transmitted to the users through either the unicast component or the broadcast component. Users are equipped with dual-system receiver capabilities so as they can receive the proposed service from either the broadcasting system or the unicast system. A minimum capacity per user, denoted as $C_{r e q}$, is required to correctly receive the proposed service. It is also assumed that the proposed service is requested by all $M$ users in the service area.

As in [3], we consider in this paper that the propagation model is limited to the effect of the path loss. A log-distance path loss model is used to evaluate the path loss value at any distance from any transmitter site. Therefore the average signalto-noise ratio (SNR) at a distance $r$ from a transmitter is given by

$$
\gamma(r)=\left(\frac{P_{e}}{P_{n}}\right)\left(\frac{\lambda}{4 \pi r}\right)^{\alpha},
$$

where $P_{e}$ the average transmission power of the transmitter is, $P_{n}$ is the average receiver noise power, $\lambda$ is the wave length related to the carrier frequency of the transmitted signal, and $\alpha$ is the path loss exponent. 


\subsection{Capacity of the broadcasting system}

In a broadcasting system, all subcarriers available for data transmissions are allocated to multiple users. It is not possible to dynamically adapt the transmission parameters according to the reception conditions of the users. Moreover, the transmissions should be configured statistically to serve the worst-case user in the service area. Therefore, the capacity of a broadcasting system is determined by the capacity of a user located at the edge of the coverage area. This implies that all users in the broadcast coverage area have the same capacity, which depends on the transmission power of the HPHT site, the targeted coverage area, and the required capacity to receive the proposed service.

In the scope of this paper, given a service capacity requirements $C_{r e q}$, the broadcasting system is planned such that

$$
B_{b c} \log _{2}\left(1+\gamma_{\text {min }}\right) \geq C_{r e q}
$$

where $B_{b c}$ is the transmission bandwidth of the broadcasting system and $\gamma_{\min }$ is the average SNR of a user located at the edge of the broadcast coverage area, i.e. at a distance $R_{\max }$ from the HPHT site.

Finally, the capacity of the broadcasting system is expressed as

$$
C_{b c}=M_{b c} B_{b c} \log _{2}\left(1+\gamma_{\min }\right),
$$

where $M_{b c}$ is the number of users that receive the service through the broadcasting system.

\subsection{Capacity of the mobile broadband system}

As shown in Fig. 1, it is assumed for simplicity that all LPLT sites have the same transmission parameters and coverage areas. Therefore, we start by focusing on a single LPLT site and then extend the results to all LPLT sites. In contrast to a broadcasting system, a mobile broadband system relies on unicast transmissions, which implies that the proposed service is delivered to each user separately. Generally speaking, a LPLT transmitter allocates a block of subcarriers, i.e. a Resource Block (RB), to a user based on the average SNR of that user. The number of RBs allocated per user depends on the resource allocation strategy used by the operator. For the purpose of this study, we consider a simple resource allocation strategy that assigns the same number of RBs to all users. In the sequel, we denote as $R B_{m}$ the number of RBs to allocate to each user $m$. To meet the service capacity requirements, $R B_{m}$ is chosen such that for all users we have

$$
B_{m} \log _{2}\left(1+\gamma\left(r_{m}\right)\right) \geq C_{r e q}
$$

where $B_{m}=R B_{m} B_{R B}$ is the transmission bandwidth allocated to user $m$ with $B_{R B}$ the bandwidth of a resource block, and $\gamma\left(r_{m}\right)$ is the SNR of a user $m$ located at a distance $r_{m}$ from the attached LPLT transmitter.

The capacity of a LPLT site is then defined as the sum-capacity of all users attached to that LPLT transmitter. However, since the number of RBs available is limited, the LPLT transmitter cannot allocate $R B_{m}$ RBs to all users. Let $\eta_{m}$ be a binary variable that indicates whether or not the transmitter has assigned $R B_{m}$ RBs to the user $m$. Finally, the capacity of a LPLT site $i$ is obtained as

$$
C_{u c, i}^{c e l l}=\sum_{m \in \mathcal{M}_{u c, i}^{c e l l}} B_{m} \log _{2}\left(1+\gamma\left(r_{m}\right)\right) \eta_{m}
$$

Where $\mathcal{M}_{u c, i}^{\text {cell }}$ is the set of users that receive the service through the unicast network from the LPLT site $i$.

\section{Performances Metrics}

To study the energy efficiency of the hybrid network model presented in this paper, we define here the service capacity and the power per bit metrics.

\subsection{The service capacity metric}

The service capacity $C_{S}$ is defined as the sum capacity of all users in the service area that have access to the proposed service. In other words, the service capacity is the sum of the capacity of the broadcasting system given by (4) and the capacity of the mobile broadband network obtained from (6). This leads to

$$
C_{S}=C_{b c}+\sum_{i \in \mathcal{N}_{L P L T}} C_{u c, i}^{c e l l},
$$

where $\mathcal{N}_{L P L T}$ is the set of LPLT cells in the given service area. The service capacity is regarded as a random variable in this paper; therefore we consider the average value $E\left[C_{S}\right]$ of the service capacity in the following. 


\subsection{The power per bit metric}

The power per bit $P_{\text {bit }}$ is defined as the total power consumption of the network divided over the service capacity. This leads to

$$
P_{b i t}=\frac{\left(P_{i n}^{b c}+\sum_{i \in \mathcal{N}_{L P L T}} P_{i n, i}^{u c}\right)}{T \times E\left[C_{S}\right]},
$$

Where $P_{i n}^{b c}$ is the total power consumption of the broadcasting system and $P_{i n, i}^{u c}$ is the total power consumption of the $i^{t h}$ LPLT cell. The total power consumptions are obtained from the EARTH project power consumption model given by (1). Finally, $T$ is the transmission time and $E\left[C_{S}\right]$ is the average service capacity.

\section{Problem Statement}

First, as stated above in Section 3.1, the planning of a broadcast network is usually done considering the worst case scenario. This planning strategy results to a low service capacity with a high transmission power of the HPHT site, which leads to high power consumption.

Second, when the number of users in a mobile broadband network increases, only a few users will receive the service since the number of available RBs is limited. This limits the number of users supported by the system.

Based on these observations, we have introduced in [6] a new mode of operation for the hybrid network where some users receive the service through the broadcast network while the others receive the same service through the mobile broadband network. This new operation mode increases the overall service capacity while guaranteeing a targeted quality of service. In this paper, we aim to extend our previous work by evaluating the energy efficiency of this new operation mode. Our goal is to find the optimal broadcast coverage area that reduces the power per bit used to deliver a service.

\section{Numerical Evaluation 6.1. Simulation settings}

Table 1 - Simulation Parameters

\begin{tabular}{ccc}
\hline \hline & Unicast & Broadcast \\
\hline Network infrastructure & LPLT & HPHT \\
Network layout & Hexagonal grid & Single cell \\
Antenna & Omnidirectional \\
Inter site distance & $1500 \mathrm{~m}$ \\
Resource allocation & $R B_{m}=5$ \\
Transmission power (EIRP) & $1.2 \mathrm{~kW}$ & $3.3 \mathrm{~kW}$ \\
Carrier frequency & $750 \mathrm{MHz}$ & $700 \mathrm{MHz}$ \\
Pathloss exponent & 2.8 & 2.6 \\
Transmission time & \multicolumn{2}{c}{$T=1 \mathrm{TTI}$} \\
Bandwidth & \multicolumn{2}{c}{$10 \mathrm{MHz}$} \\
\hline \hline
\end{tabular}

We consider the delivery of one high definition (HD) linear service to $M$ outdoors users in a $30 \times 30 \mathrm{~km}^{2}$ service area. The $M$ users are uniformly distributed in the service area. The minimum capacity $C_{r e q}$ required to receive the service is $C_{\text {req }}=2$ Mbps.

For the mobile broadband network, we consider a LTE unicast system. The smallest radio resource unit that is allocated to a user is the LTE physical resources block (PRB) which is a group of 12 subcarriers of $15 \mathrm{KHz}$. The total bandwidth is divided in subchannels of $180 \mathrm{KHz}$, i.e. $1 \mathrm{PRB}$. The length of one PRB is $0.5 \mathrm{~ms}$ which is the length of a slot. A slot is composed of 7 OFDM symbols. The resource allocation is done in a time and frequency domain at each subframe. A subframe is composed of 2 slots, which forms a Transmission Time Interval (TTI). In this paper, a simple resource allocation strategy is assumed for the unicast network; therefore from (5) the number of RBs assigned to a user is fixed to $R B_{m}=5$.

For the broadcast network, we consider a dedicated LTE broadcast system, where all PRBs are assigned to all users covered by the broadcast network. This assumption is made to highlight the benefits of broadcast and unicast cooperation 
without any physical layer comparison. Further, this assumption allows us to simulate the offloading cooperation scenario where the LTE broadcast mode (e.g. eMBMS) is improved and then embedded in DVB-T2 future extension frame as suggested in [9], [10]. Finally, from (3) the minimum SNR is set to $\gamma_{\min }=0[d B]$.

Radio network simulations have been performed with the Vienna LTE-A downlink system level simulator [11]. Numerical results were provided by emulating the behavior of different hybrid operation modes. The simulator functionalities have been extended to integrate HPHT transmitters operating in a broadcast mode using a dedicated LTE broadcast signal such as the eMBMS++ [9], [10]. Therefore, typical values of LTE system parameters listed in Table 1 are considered for numerical evaluations. To obtain statistically consistent results, we have performed Monte Carlo simulations to evaluate the metrics presented in Section 3.

\subsection{Results and Discussions}

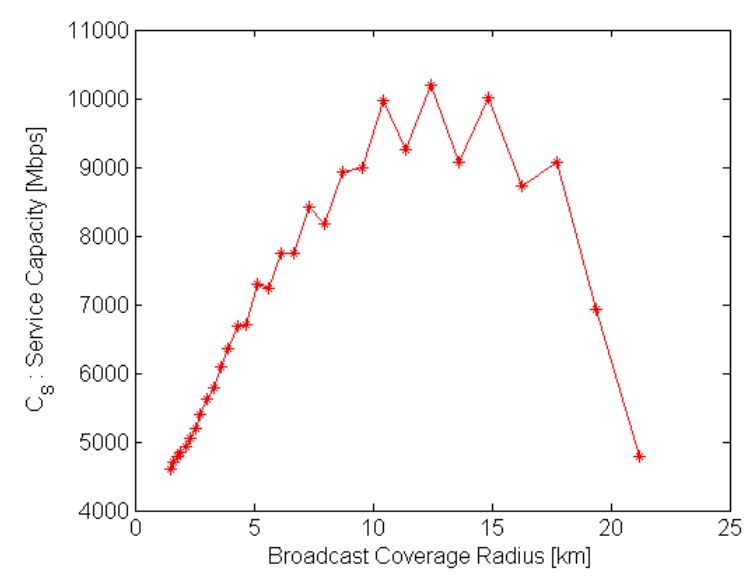

(a)

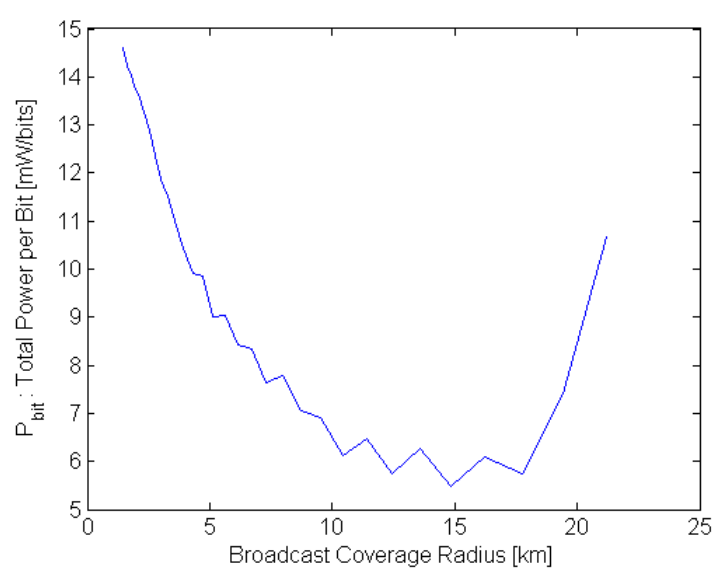

(b)

Fig. 2 -Evolution of the service capacity (a), and the total power per bit (b) vs the broadcast coverage radius. Results are presented for $M=1000$ UEs in the service area.

Fig. 2(a) shows the evolution of the average service capacity of the hybrid network as a function of the broadcast coverage area in terms of the coverage radius. The results are presented for 1000 UEs. First observe that the lowest service capacity is achieved without network cooperation, i.e. when both networks are used in a standalone mode. Furthermore, Fig. 2(a) also shows that the service capacity is improved with network cooperation. The maximum service capacity is reached when the broadcast coverage radius is reduced to $12 \mathrm{~km}$, which is the optimal broadcast coverage radius in this situation. The results suggest that network cooperation increases the average service capacity.

Fig. 2(b) gives the evolution of the average total power per bit as a function of the broadcast coverage radius for 1000 UEs in the service area. The results show that the power per bit used is higher when there is no cooperation. On the contrary, with network cooperation, the total power per bit is significantly reduced. As expected, Fig. 2(b) shows that increasing the service capacity reduces the total power per bit used (please refer to (8)). In addition, Fig. 2(b) also shows that for 1000 UEs, the minimum power per bit is achieved when the broadcast coverage radius is set to $15 \mathrm{~km}$, which leads to a new optimal broadcast coverage radius. Observe that this optimal broadcast coverage radius that leads to the reduction of the total power per bit used does not match the optimal broadcast coverage radius that leads to the maximization of the service capacity. Therefore a tradeoff between service capacity and total power per bit used has to be made. Nevertheless, the service capacity and the total power per bit are both improved with such hybrid approaches.

Finally the irregularities observed on Fig. 2(a) and Fig. 2(b) are related to the SINR-to-capacity mapping function used by the simulator. Indeed, the function can map several SINR values to the same capacity.

\section{Conclusion}

This study has investigated the energy efficiency of using network cooperation to deliver linear services to mobile and portable devices. We have proposed new hybrid operation modes to optimize the overall capacity of the hybrid network while reducing the power per bit used to deliver the proposed service. The results suggest also that a trade-off between the maximization of the capacity and the minimization of the total power per bit used has to be made. Both optimal operation modes cannot be reached at the same time. 


\section{References}

[1] D. Catrein, J. Huschke, and U. Horn, "Analytic evaluation of a hybrid broadcast-unicast TV offering," in IEEE VTC Spring, 2008, pp. 2864-2868.

[2] C. Heuck, "An Analytical Approach for Performance Evaluation of Hybrid (Broadcast/Mobile) Networks," IEEE Trans. Broadcast., vol. 56, no. 1, pp. 9-18, Mar. 2010.

[3] N. Cornillet, M. Crussiere, and J.-F. Hélard, "Optimization of the energy efficiency of a hybrid broadcast/unicast network," in IEEE WCNCW, 2013, pp. 39-44.

[4] A. A. Razzac, S. E. Elayoubi, T. Chahed, and B. El Hassan, "Planning of Mobile TV service in standalone and cooperative DVB-NGH and LTE networks," in WiOpt, 2013, pp. 609-614.

[5] A. A. Razzac, S. E. Elayoubi, T. Chahed, and B. El-Hassan, "Comparison of LTE eMBMS and DVB-NGH mobile TV solutions from an energy consumption perspective," in IEEE PIMRC Workshops, 2013, pp. 16-20.

[6] P.-A. Fam, M. Crussière, Jean-François Hélard, P. Bretillon, and S. Paquelet, "Global Throughput Maximization of a Hybrid Unicast-Broadcast Network for Linear Services," in ISWCS'2015 - Track 1- PHY, 2015, pp. 146-150.

[7] G. Auer, O. Blume, V. Giannini, I. G. ETH, M. A. Imran, Y. J. EAB, E. Katranaras, M. O. EAB, D. S. TI, P. S. EAB, and others, "INFSO-ICT-247733 EARTH."

[8] C. Desset, B. Debaillie, V. Giannini, A. Fehske, G. Auer, H. Holtkamp, W. Wajda, D. Sabella, F. Richter, M. J. Gonzalez, H. Klessig, I. Godor, M. Olsson, M. A. Imran, A. Ambrosy, and O. Blume, "Flexible power modeling of LTE base stations," in IEEE Wireless Communications and Networking Conference (WCNC), 2012, pp. 2858-2862.

[9] M. Crussiere, C. Douillard, C. Gallard, M. Le Bot, B. Ros, A. Bouttier, and A. Untersee, "A Unified Broadcast Layer for Horizon 2020 Delivery of Multimedia Services," IEEE Trans. Broadcast., vol. 60, no. 2, pp. 193-207, Jun. 2014.

[10] D. Rother, S. Ilsen, and F. Juretzek, "A Software Defined Radio based implementation of the 'Tower Overlay over LTE-A+' system," in IEEE Broadband Multimedia Systems and Broadcasting (BMSB), 2014, pp. 1-6.

[11] M. Taranetz, T. Blazek, T. Kropfreiter, M. K. Muller, S. Schwarz, and M. Rupp, "Runtime Precoding: Enabling Multipoint Transmission in LTE-Advanced System-Level Simulations," IEEE Access, vol. 3, pp. 725-736, 2015. 